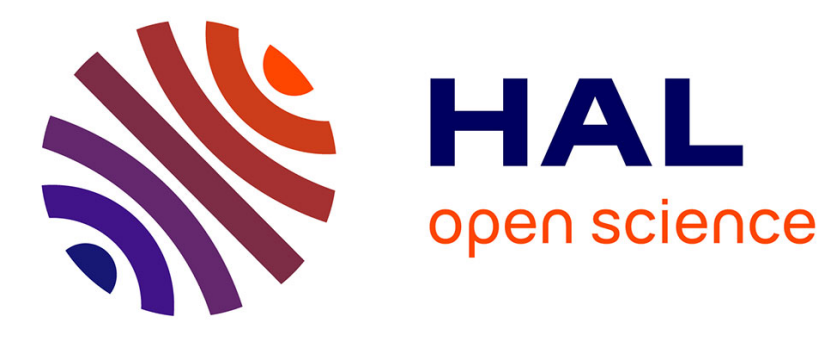

\title{
The reasonable effectiveness of Mathematics and its Cognitive roots
}

\author{
Giuseppe Longo
}

\section{To cite this version:}

Giuseppe Longo. The reasonable effectiveness of Mathematics and its Cognitive roots. Boi, Luciano. Geometries of Nature, Living Systems and Human Cognition. New Interactions of Mathematics with Natural Sciences and Humanities, World Scientific Publishing, pp.351-382, 2005, 978-9812564740. 10.1142/9789812700889_0007. hal-03319097

\section{HAL Id: hal-03319097 https://hal-ens.archives-ouvertes.fr/hal-03319097}

Submitted on 11 Aug 2021

HAL is a multi-disciplinary open access archive for the deposit and dissemination of scientific research documents, whether they are published or not. The documents may come from teaching and research institutions in France or abroad, or from public or private research centers.
L'archive ouverte pluridisciplinaire HAL, est destinée au dépôt et à la diffusion de documents scientifiques de niveau recherche, publiés ou non, émanant des établissements d'enseignement et de recherche français ou étrangers, des laboratoires publics ou privés. 


\title{
The reasonable effectiveness of Mathematics and its Cognitive roots $^{1}$
}

\author{
Giuseppe Longo \\ Laboratoire d'Informatique \\ CNRS et Ecole Normale Supérieure \\ 45, Rue D'Ulm \\ 75005 Paris (France) \\ http://www.di.ens.fr/users/longo
}

"At the beginning, Nature set up matters its own way and, later, it constructed human intelligence in such a way that [this intelligence] could understand it" [Galileo Galilei, 1632 (Opere, p. 298)].

"The applicability of our science [mathematics] seems then as a symptom of its rooting, not as a measure of its value. Mathematics, as a tree which freely develops his top, draws its strength by the thousands roots in a ground of intuitions of real representations; it would be disastrous to cut them off, in view of a short-sided utilitarism, or to uproot them from the ground from which they rose" $[\mathrm{H}$. Weyl, 1910].

Summary. Mathematics stems out from our ways of making the world intelligible by its peculiar conceptual stability and unity; we invented it and used it to single out key regularities of space and language. This is exemplified and summarised below in references to the main foundational approaches to Mathematics, as proposed in the last 150 years. Its unity is also stressed: in this paper, Mathematics is viewed as a "three dimensional

1 In Geometries of Nature, Living Systems and Human Cognition, a series in "New Interactions of Mathematics with Natural Sciences and the Humanities" (L. Boi ed.), pp. 351 382, World Scientific, 2005. 
manifold" grounded on logic, formalisms and invariants of space; we will appreciate by this both its autonomous generative nature and its effectiveness. But effectiveness is also due to the fact that we re-construct the world by Mathematics: we organise knowledge of space and language by Mathematics, and give meaning by it to their structuring. But, what is "meaning", for us living and historical beings? What does "mathematical intuition" refer to? We will try to propose an understanding of these crucial aspects of the mathematical praxis, often disregarded as "magic" or as beyond any scientific analysis. Finally, some limits of the remarkable, but reasonable effectiveness of Mathematics will be sketched, in particular in reference to its applications in Biology and in human cognition

\section{Introduction}

In this century, two major foundational paradigms have been splitting man and the world around him from one of its major conceptual constructions, Mathematics. On one side, formalism proposed the perfect rigor of mechanical rules as the core for certainty, effectiveness and objectivity of Mathematics: stepwise deductions along finite strings of symbols, perfectly independent of meaning, were meant to reconstruct completely mathematical reasoning or even propose a method for mathematical creativity (to be transferred, possibly, into digital computers). The philosophical background (and the practical aim, in the latter case - Artificial Intelligence) was based on the idea of a possible mechanical implementation of "the Universal Laws of Thought": once these were all formally described in a symbolic notation, without the ambiguities of meaning, we could fed a machine with them and completely simulate reasoning, action or general human behaviour.

On the other hand, the naive platonistic reactions to this bold program, explained the failure of the formalist program as well as the certainty and objectivity of Mathematics by assuming independent ontologies; usually, this perspective as well refers to absolute "laws of thought" or to the perfect geometry of absolute "mathematical spaces", but as meaningful structures of truth, as "essence" which underlies the world, "per se".

In either cases, Mathematics was separated from our "being in the world", from our forms of knowledge as embedded in our living and historical beings, where "understanding" is based on non-arbitrary proposal and descriptions constructed while interacting with that very world. Then, time to time, some leading colleagues came up with exclamations of surprise: "how is it possible that this game of meaningless symbols (or, alternatively, this perfectly independent ontologies) happens to say something, indeed a lot, about the "concrete reality" surrounding us? What an amazing miracle!". So, after inventing or accepting a schizophrenic split, some tried to recompose it by referring to magic or metaphysical, inexplicable connections (even ... "not deserved"?!, see [Wigner, 1960]) and 
forgot that human knowledge should be analysed in a scientific perspective and not in terms of miracles nor in search for "absolute knowledge".

Mathematics is the result of a "knowledge process", as one of the ways we relate to the world, while constructing our own "cognitive ego": our intelligence is an ongoing and active organisation of phenomena while trying to make them intelligible to us, it is co-constructed while structuring phenomena. We cannot separate Mathematics from the understanding of reality itself; even its autonomous, "autogenerative" parts, are grounded on key regularities of the world, the regularities "we see" and develop by language and gestures.

In this paper we will try to hint to the unity of human conceptual constructions, as well as to the rich way in which various forms of knowledge are articulated. They constitute a network of meaningful attempts to understand the world, all rooted in "acts of experience", as active forms of interpretation and reconstruction of reality, deeply embedded in our cognitive and historical lives. Mathematics is one of these forms whose peculiar nature, by its "conceptual invariance and stability" as we will stress, is not independent from the others and, by this, it can "say something", indeed a lot - but not "everything" - about them and the world: it is reasonably effective.

\section{The logicist and formalist foundation. The role of "space".}

\subsection{From the Geometry of space to Logical Truth}

Let's first briefly recall a story which begins with a major crisis: the loss of certainty in the absolute of physical space, whose geometry was identified to Euclidean geometry. For more than two thousands years, the "pure intuition of space per se" guaranteed certainty and foundation to the many constructions of Mathematics: the "synthetic a priori" of geometry was behind the objectiveness and effectiveness of the drawing of the geometric sign, of the proof carried on "more geometrico", on the perfect planes and spaces of Euclidean geometry. But ... what happens if the world were curved?

With this motivation, Gauss analysed another "possible world": the idea was to look at a surface "per se", not as embedded in an Euclidean space. That is, to analyse the surface "without leaving it", by moving, say, along its geodetics, as lines of (relative) minimal length. The connection to Euclid's fifth axiom was explicitly made by Lobachevskij and Bolyai. Riemann pursued Gauss's aims and developed the (differential) geometry of curved surfaces: he thus invented the fully general notion of "manifold" (topological, metric, differentiable manifolds... in today's terminology). Riemann actually considered the curvature of the physical space as related to the presence of physical bodies ("their cohesive forces", as said in his Habilitation): the notion of Cartesian dimension, a "global" property 
of space, is topological, while the curvature and the metric is a local property, which may depend on the "cohesive forces of matter", an amazing anticipation of Einstein's relativity 2. As a matter of fact, by the analysis of the geometric structure of space (as ether), Riemann meant to unify action at distance (heath, light ... gravitation).

Clearly, Riemann greatly contributed, by his n-dimensional differential geometry, to demolish the absolute and certainty of Euclidean space, just a special case of his general approach. Yet, he tried to re-establish knowledge as related to our understanding of the (physical) world. For him, geometry is not "a priori" by its axioms, but it is its grounded on certain regularities of physical space, to be singled out and which have an objective, physical, meaning (continuity, connectivity and isotropy, for example). In Riemann's approach, we actively structure space, as manifold, by focusing on some key properties, which we evidentiate by "adding hypotheses". Thus the foundational analysis consists, for Riemann, Klein, Clifford, Helmoltz ... in making these regularities explicit and in spelling out the transformations which preserve them.

However, this "relativized" neo-kantian attitude turned out to be unsatisfactory for many, since it dangerously involved an analysis of the "genesis of concepts", as apparently originating from our more or less subjective presence in the physical world. In fact, these concepts, as for instance those of differential form, group and curvature are objective because they are invariants that we actively single out of the hysical world; that is, they become concepts as a result of the interaction, at the phenomenal level, between us and the world. How to re-establish then absolute certainty and objectiveness, after the shocking revolution of non-Euclidean geometries, while avoiding this "implication of the subject" into knowledge?

First, avoid any reference to space and time, the very reference that had given certainty, for so long, not only to geometry, but also to algebra and analysis. For Descartes and Gauss, say, algebraic equations or the imaginary numbers are "understood" in space: this is so in analytic geometry and in Argand-Gauss interpretation, over the Cartesian plane, of $\sqrt{ }-1$ (of the complex numbers, thus). But, if our relation to space is left aside in order to avoid the uncertainties of the "many geometries" and the shaky sands of human cognition, then language remains, in particular the logical laws of thought that the English school of algebra had already been putting forward, in a minimal language of signs ([Boole,1854]). Language, considered as the locus of the manipulation of (logical) symbols, with no reference to phenomenal space, nor, in general, to forms of experience.

Frege best represents this turning point, in the foundation of Mathematics. His search

2 Riemann's Habilitation is of 1854, under Gauss' supervision [Riemann, 1854; it. transl. 1999]; more remarks were made in the '60s. A broad analysis may be found in [Boi, 1995], [Tazzioli, 2000]. 
for "absolute knowledge" was meant to exclude, first, any hint to "intuition" or "empirical evidence", any analysis of the "knowledge process" (see [Frege,1884]), in particular in reference to phenomenal space and time. In his work, the fight against Stuart-Mill (empiricism) is explicit, the one against Herbart (psychologism), who had largely influenced Riemann, and to Riemann himself is evident: empiricism and psychologism are Frege's worst enemies.

We must acknowledge the depth of Frege's analysis. His scientifically plane style as well: he established a novel standard of rigor by his "language of formulae", where universal and existential quantification (the "for all $\mathrm{x}$..." and "there exists $\mathrm{x}$..." so relevant in Mathematics) are finally handled in a uniform and sound way, in contrast to the mathematical practice up to that time, or most of it (the formal gaps in Cauchy's work, say, are well-known). The too elementary "laws of thought" of the British algebraists (Boole, Babbage) are thus enriched by the so-called (first order) quantification: Mathematical Logic is born, as the search for "unshakeable certainties" in absolute assumptions and laws of deduction. Absolute, but meaningful: the assumptions and the laws must have a "logical meaning"; even arithmetical induction, a technical proof principle for number theory, has a logical meaning for Frege. Arithmetical computations are logically valid deductions. The reference is again to a platonic realm of logical and absolute truth, independent of man: "pure concepts", without conceptor.

The search for foundation (and certainties) in the interaction between us and the world, starting with physical space, is abandoned: Mathematics is brought back to sit in platonic realms, detached from us and the world. What a surprise when some will rediscover that it is very ("unreasonably") effective, in Physics in particular, as if it where constructed to say something about the world, in a suitable language for us.

\subsection{Formalism and linguistic stratifications.}

An alternative path towards grounding Mathematics away from human reasoning about reality, was proposed by Hilbert's foundational work, once again for good reasons and by a strong proposal.

Suppose that you have a proof of existence and, maybe, of uniqueness of the solution of a system of (differential) equations or of a finite basis for certain algebraic systems; yet, assume that you cannot give the solution explicitly, as an elementary function, say, nor construct it as the limit of suitable (Fourier) series nor provide effectively the finite basis. Where does this solution, this finite set, live? In which platonic/logical realm?

Hilbert has an original and robust proposal for an answer to this: provide a finite axiomatic frame for your proof, with finitary (effective) deduction rules, then the "existence property" in your theorem may be guaranteed by a proof of consistency (non-contradiction) 
of the axiomatic theory. In '900, he poses, as a key open problem, the proof of consistency of Arithmetic (and thus, by Cantor-Dedekind construction of the reals, of Analysis). A proof to be carried on in a "potentially mechanizable" fashion, in order to reduce certainty to the finite manipulation of symbols, with no reference to (actual) infinite nor to (possibly geometric) meaning. This is Hilbert's conjecture of the finitistically provable consistency of Arithmetic (and other key formalised theories).

The project is strong and revolutionary: two dangers are avoided at once. Infinitesimal analysis had introduced the infinite to analyse the finite: since the XVIII century, powerful tools had been developed to describe physical, finite, movement around us (speed, acceleration) by actual limits, some sort of dangerous metaphysical infinities (based on Leibniz's monads, say). In spite of the work of Cantor, the foundation of infinity remained uncertain and flawed by paradoxes (contradictions). By the formalist program, and its developments, the situation could be reversed: in particular, once proved, by finitistic tools, the consistency of theories that formalise the infinite, such as (Zermelo's) Set Theory, then, by the consistency of the theory, the "existence" issue is solved, including the existence of infinite objects (sets, functions, actual limits ...). As a matter of fact, in Hilbert's program, mathematical existence is provable consistency of the intended theory, and nothing else. No need to dream of platonic realms, at least in the foundational work: once consistency is proved by finitary tools, the working mathematician could happily live in Cantor's paradise of infinite and ideal objects (in his practice of mathematics, Hilbert was far from being a formalist!). Moreover, the shaky reference to space (Euclidean, non-Euclidean? physical?) may be avoided as well: as for foundational purposes, geometries are just finite sets of (provably consistent) formal axioms, which may be interpreted in many ways, and the interpretations are irrelevant to deductions and proofs. And this is so, since deduction rules are applied mechanically, i.e. according only to the syntactic structure of the formulae (well-formed strings of symbols) in the assumption, with no reference to their meaning (logical, geometric ...).

Then the bold enterprise of the formalist finitism began, grounded on one further and crucial idea of Hilbert's. He proposes to carry on the foundational analysis in a mathematised "metalanguage", whose object of study is the object language of the formal theories. Moreover, these theories, at their purely syntactical level, with no reference to metalanguage nor meaning, should be able to describe completely Mathematics. That is, any formalised assertion of it should be decided by finitistic deductions from the axioms. And here is Hilbert's conjecture of "completeness" of the key axiomatic theories, such as formal arithmetic. In summary, by finitistic metamathematical tools one should be able to prove consistency and completeness of the core of Mathematics.

Hilbert's "linguistic stratification" (language, meta-language) is a remarkable way to 
organise the "discourse of Mathematics", perhaps comparable to Euclid's proposal to organise physical space. Yet, as much as Euclid's, this is not an absolute. That is, Hilbert's proposed distinction between theory and metatheory is not the only frame within which one may approach the foundational problems: other conceptual construction may violate this organisation and the blend of levels (and of meanings) may require an analysis that goes well beyond it. Indeed, the failure of this paradigm is the very reason for the incompleteness phenomena, as we will briefly hint below. The fact is that the formalist paradigm for mathematical knowledge (both foundation and praxis) marked the century and many still beleive that a "sufficient collection of (set-theoretic?) axioms", once fully formalised, may allow a complete deduction of Mathematics ... yet, this very same people show a great surprise when, in spite of this assumed automatism (or "meaning independence") of the discipline, it helps us in understanding and giving meaning to the world.

A few soon reacted to Hilbert's program, such as the "lone wolf" among Hilbert's students, Hermann Weyl, who conjectured in Das Kontinuum, 1918 (!), the incompleteness of formal arithmetic ( $\$ .3$ in [Weyl,1918]). He also stressed in several places that the idea of mechanisation of Mathematics trivialises it and misses the reference to meaning and structures. Unfortunately, Weyl calls this crucial reference to meaning, "the mathematician's insight" or "intuition", with no further explanation; a reference to the "unspeakable" that we have to overcome: below, we will try to understand what these words may mean. Besides Weyl (and Poincaré and a few others), Wittgenstein is another thinker who criticised Hilbert's program. For him "Hilbert's metamathematics will turn out to be a disguised Mathematics" [Waismann, 1931], since "[A metamathematical proof] should be based on entirely different principles w.r. t. those of the proof of a proposition ... in no essential way there may exist a meta-mathematics" (see Wittgenstein, Philo. Rem., $\S 153$; quoted in [Shanker,1988]), and ... "I may play chess according to certain rules. But I may also invent a game where I play with the rules themselves. The pieces of the game are then the rules of chess and the rules of the game are, say, the rules of logic. In this case, I have yet another game, not a metagame" [Wittgenstein, 1968; p. 319].

As for arithmetic, the key theory for finitistic foundationalism, these remarks may be understood now in the light of Gödel's Representation Lemma [Gödel, 1931]: by this very technical result, one may encode the metatheory of arithmetic into arithmetic itself, thus the "rules of the metagame" are just viewed as ... rules of the "arithmetical game". Moreover, many proofs which entail the consistency of arithmetic, such as (Tait-)Girard proof of "normalisation" of Impredicative Type Theory ([Girard et al., 1989]), need a blend of metalanguage and language; or even purely combinatorial statements, such as Friedman's Finite Form of Kruskal's theorem, provably require the same entangled use of metatheory, 
theory and semantics, by the impredicative notions involved: an indirect confirmation of Wittgenstein's philosophical insight (see [Harrington et al., 1985] for the mathematics; a discussion and more references are in [Longo, 1999a]). These theorems are some of the many recent examples of more or less "concrete" incompleteness results of formal arithmetic; that is, they are interesting arithmetic statements whose proof requires essentially "non formalizable" (not effective-axiomatic) tools. They are not self-referential "tricks" such as Gödel's independent statement of arithmetic, a simple proof-theoretic translation of the Liar's Paradox ${ }^{3}$.

The point is that, in the arithmetic proofs of these interesting (concrete) statements, meaning is essential; more precisely, at a certain point of the proof, in order to go "from one line to the next", one has to refer to some variables as interpreted by sets, to others as interpreted by their elements (use of some form of an impredicative second order comprehension axiom), or to the well-ordering of integer numbers (second order induction), or to similar "concepts" which provably cannot be formalised arithmetic, in a finitistic way. Computers get stuck, but human beings, by referring to the well-ordered structure of integer numbers in space or time, by "seeing" sets and elements, as meaningful and conceptually distinguished notions, have no problem in understanding and carrying on the proof, even though these are provably not formalizable in a finitistic, mechanisable fashion (or by symbols which run independently of meaning). Thus, meaning steps in and the formalist analysis has been such a strong paradigm as to prove this for us, by these and the many other unprovability results in formal theories (the independence of the "continuum hypothesis" and of the "axiom of choice", whose analysis motivated formal Set Theory are further examples, see any text in Set Theory or [Longo, 1999c] for more discussions and references to this even more severe "debacle" of formalism).

It is time now to go further and stop believing in the absolute of the Hilbert(-Tarski) foundational frame (theory, metatheory and semantics); that the finitistic formal level and the metalinguistic, yet mathematical, analysis can say everything about Mathematics and its foundation or that the close structuring of mathematical concepts, in this specific way, coincides with the foundational analysis of Mathematics. A large amount of technical work can surely still be done along this paradigm, as reductions to least purely formal frames are often very informative; it is the underlying philosophy that must be overcome. Many theorems tell us that this failed, yet the philosophical prejudice, the unscientific myth of "absolute knowledge" or the reference to certainty as mechanical deduction only or the idea that the foundational issues of Mathematics may be treated only mathematically, still resists,

3 Gödel's incompleteness theorem is an immense achievement as for technical inventions and insights, essentially by the tools used in the proof of the Representation Lemma: gödelization, recursive functions ... . The "unprovable" statement "per se" has no mathematical interest, in contrast to the many recent examples, as those quoted above. 
along the lines of Hilbert's relevant, but too rigid stratification, not proper to the humanmathematical experience. The major merit of the formalist-mechanicist approach was to set the philosophical and mathematical basis for inventing, in the thirties, the (mathematics of) computing machinery: Boole-Frege "laws of thought", independent of human thinking, are finally implemented in meaningless strings of symbols, pushed by machines according to meaningless rules. The idea of transferring human rationality in machines, to refer to computers as the paradigm for "logic and rigor", is probably the main fall-out (indeed, a relevant one) of this "splitting" proposal: splitting man from one of his major form of knowledge, Mathematics.

Yet, as a consequence of some of the incompleteness theorems mentioned above, the concept of infinity turns out to be essential to prove the consistency of formal arithmetic; let alone of those theories of infinity, the theories of sets whose consistency depends, at each infinitary level, by the use of even more infinitary constructions. Now, infinity is a robust human conceptual construction, the object of a lively debate for centuries, which stabilised with Cantor into a operational, mathematical notion. As suggested in [Longo, 1999a], we have, so far, no better "foundation" for the infinite than the reference to its historically meaningful specification as a mathematical concept, i.e., the analysis of its "progressive conceptualisation", to put it in F. Enriques' terms. The set theoretic specification of this concept, a technically remarkable clarification and "stabilisation" of the notion, is not a "foundation", since it transfers to larger infinities, by the consistency proofs, the foundation of each level of infinity. The foundation of mathematical infinity lies in the analysis of its conceptual genesis, of the knowledge process which brought it to stabilise as a mathematical invariant (see [Longo,2001]). The rooting of this path in our relation to the world, its constitutive role for our (mathematical) interpretation and re-construction of it and, thus, its meaning, are the reasons of its mathematical effectiveness, as a conceptual tool of analysis. In particular, the use of the concept of infinity is robust and effective because it is codefined with our ways of organising the world by Mathematics: from the early "theological" debates, to the structuring of trajectories and lines, by tangents and limits (Newton), rich of physical meaning (speed, acceleration ...). These notions organise, for us humans, the movements of finite objects around us by actual infinity and, once distilled in a rigorous mathematical practice (since Cantor), they are as effective as no other human construction in describing physical reality.

\subsection{Mathematics as a "three dimensional manifold".}

In a very schematic way, I tried to summarise the main approaches to the foundations of Mathematics by a very incomplete reference to three major scientific personalities: Riemann, Frege and Hilbert. Each one of their intended foundational ways stresses some crucial 
aspects of the conceptual construction of Mathematics. As a matter of fact, there is no doubt that Mathematics is grounded in logic, those "if ... then ... else ..." and much more that unfold along proofs (Frege). Similarly, one uses formal computations, in an essential way: purely algebraic reasoning pervade proofs and equations follow from equations following abstract rules, whose meaning is irrelevant to deductions (Hilbert). But symmetries also or other regularities of space (connectivity, say) contribute in singling our structures and their relations (Riemann). These have no logical meaning, yet they appear in theory building and in proofs (see for this the novel insight which originated in [Girard,1987]).

This richness of Mathematics is lost in the logicist and formalist approaches: only logic or finitist formalism (and possibly not both!) found Mathematics. In particular, our relation to space is only a matter of "ad hoc extensions", largely conventional for the formalist, to be found on the concept of "ratio as number" for the logicist [Frege,1884; pp. 56-57 and $\S .14]^{4}$.

One may instead synthesise the variety of grounding components of Mathematics by looking at it as to a "three dimensional manifold" (a generalized "three dimensional space"). Mathematics is found on, and uses in its developements:

- logic,

- formal computations,

- geometric construction principles.

The generative nature of Mathematics is due exactly to the blend of these "three dimensions". For example, once some key regularities of space are singled out (by suitable linguistic descriptions), one uses logical or formal principles (which belong to language) to derive new properties of space (to be given in language); similarly, but entirely within language, formal computations unfold consequences of logical principles. In either case, invariance preserving transformations are applied, as both the rule of logic and of formal computations preserve meaning as invariant or stable conceptual structures: this is what they have been spelled out for! (technically: they preserve "validity w.r.t. the intended interpretation"). In particular, they preserve meaning in space. That is, if a statement about space, say, is realized in some structuring of it, then logic and computations preserve its validity via deductions and lead us to new realizable statements about the world. Thus, we add the generative power of (logical or formal) reasoning, a key linguistic tool for organising/understanding the world, a "meaning preserving" tool, and transform basic invariants of space, say, into novel meaningful invariants. Sometimes this is done by taking

4 This is essentially insufficient: only Euclidean geometry is closed under homotheties - or only its group of automorphisms includes ratio preserving maps; even in the '20s Frege will keep referring to Euclidean geometry only, in his attempt to broaden the logicist foundation of Mathematics. 
major detours and, then, remote techniques for algebra turn out to be useful in Geometry or in differential calculi or alike.

But one may also go from (formal) language to space. For example, by symmetries and dualities one "understands", in the Cartesian plane, the "meaningless" $i=\sqrt{ }-1$ and even $-i$ (by a key generative simmetry of the Cartesian plane: the addition of the negative coordinates). And then the complex numbers, the result of the formal/mechanical solutions of certain equations, suddenly become particularly relevant for Physics, that very Physics which describes space and action in space: formal matrices, say, represent generalised vectorial computations in multidimensional spaces ... (a crucial step in Quantum Mechanics). Then one derives properties of complex numbers, and of functions on them, by a blend of spatial properties (symmetries ...) and language transformations, i.e. by geometry, logic and algebra.

There is no miracle here, but the relevance of a conceptual construction, Mathematics, whose aim is to focus on key invariances: of space, of reasoning and of formal deduction. Geometry makes space intelligible by singling out some key regularities of it and turning them into invariant properties w.r.t. to the intended transformations that action in space suggest to us. Similarly, logic evidentiate some invariants of language: the logical principles "pass through proofs" or are present on all proofs and do not depend on contextual constructions. Some may be detached from meaning, even logical meaning, and become purely formal computations, to be applied mechanically: the formal rules then impose the computational invariants.

The blend of these three conceptual dimensions makes Mathematics generative and effective. This form of generativity is the reason of the "extraordinary" effectiveness of mathematics.

In summary, starting with some basic regularities (invariants) of space, say, Mathematics "generates" further invariant properties by using logical trasformations and invariants (regularities) of language (that very language we use to "organise" space) and so on so forth in all possible combinations of its "three dimensional" nature (in Girard's logical systems, for example, one uses geometric principles - such as symmetries, connectivity, ... the unfolding of knots in "proofs nets" - to carry on deductions). This is effective, as its strength is in the maximal (not absolute!) invariance and stability of each dimension of the conceptual constructions and on its "interpretation preserving transformations". It may be surprising, because, say, the unexpected spatial interpretation of the formal symbol $i$ embeds it into a novel meaningful frame, the locus for deductions grounded on very different principles, space, yet compatible as given in another conceptual dimension. 


\section{Meaning and Intuition}

The foundational project, besides the formalist analysis that can still provide relevant information on least deductive frames (and suggest ways to implement on computers as much Mathematics as possible, an interactive help to the proof), should now be extended to an analysis of "meaning" and "intuition", this betrayed notion by the logicist and formalist tradition and that many, in contrast, mentioned so often (Riemann, Poincare', Weyl ...). The point is that Mathematics is effective also because it is meaningful and because it is grounded in intuition. Mathematical knowledge is constructed by an interaction with human intuition, a notion to be discussed below, which is dynamically modified along the genesis of the discipline.

As for meaning, before proposing a further specification, in connection to intentionality and life (in §. 3), let's more closely describe meaning as given by a network of (sufficiently stable or invariant) practical and conceptual experiences, grounded on mathematical but also on other (conceptual) praxis. For example, meaning is added to an analytic equation of geometry, when it is understood as a line, a plane, a n-dimensional surface $\ldots \sqrt{ }-1$ aquires a meaning on the cartesian plane, as a different conceptual constructions, "per se". Thus, meaning is first in "setting bridges", displaying metaphors, elucidating rigourously references, by mutual explanations. The very fact that this embedding and bridging is made in many and different active experiences allows us to extract the meaningful mathematical invariants, the stable "conceptual contours" common to many of them.

Then, intuition may be analysed as a direct, yet pre-conceptual, reference to a variety of meanings, whose interconnections motivate and provide robustness to the mathematical construction. It is a largely pre-linguistic experience; often an "insight" or "mental seeing" of (part of) the mathematical structures, as mental constructions, whose network is meaningful to us. The mathematical intuition is the ability to insert a more or less formal expression, either a "hint" or a symbolic notation, into a "network" of meanings. Seing is its main organ, as the trained mathematician can (re-)construct an image from formulae, similarly as a trained musician may "hear" music when reading a piano score (the mental reconstructions of images from verbal descriptions is a very common experience). Thus, intuition precedes and follows language. It follows it, in the sense that it is "seing" the result of a conceptual, even formal, construction, also or entirely developped in language; it precedes it, as this seing, usually, needs to be later specified in language, as it may yield a vision of a novel structure, a combination of previously inexisting ones, to be fully determined and communicated by language.

Mathematical intuition is far from being static and "pure". Training is an essential part of it: it is ever changing and rich of the impurities of the subjective experience, yet it is brought to be shared and "objective" by the common cognitive roots and by intersubjective 
exchange. It is a crucial part of the good teaching of mathematics, it really makes the difference w.r.t. the bad teaching, as teaching to use mechanically "compulsory", meaningless formulae: the good, passionate mathematician teaches "to see" and to conjecture (seing before the proof), before and jointly to teaching how to prove. Intuition is mostly "local", as pointed out in [Piazza, 2000], even if it may be very broad (yet, "if everything were intuitable, nothing would realy be so" [Piazza, 2000]).

The mathematician "understands" by using well established or original references to meanings and structures: he then "sees" the meaning as a structure (geometric, algebraic) or in a structure, this is his intuition. Intuition integrates different conceptual experiences, it allows a blend of methods without caring of the details of a formal proof: one "sees" unrelated structures toghether, proposes unexpeted bridges, by joining, in a new meaningful structure, long lasting work in different areas.

This constituting of meaning should not be understood in a shallow way: its analysis must span from the earliest and deepest relation of our "being" in the world and relating to others, even in pre-human phases, up to the human, historical and most complex endeavour towards knowledge.

The project then is to single out the objective elements of this formation of meaning and how it underlies intuition, since they step in the proof, as we said. The aim is to analyse in a scientific fashion what has been explicitly "hidden under the carpet" along the XX century: the role of intuition and meaning, even in proofs.

Memory, for example, is one of the ways by which we constitute "meaningful" invariants, by selecting, comparing, unifying, by providing analogies. Forgetting is one of the major tasks of human memory, in contrast to digital data base: a goal directed or "intentional" choice, yet largely unconscious, of what is relatively relevant, a way to focus or single our what is stable or what is worth considering as an invariant. And by memory I both refer to individual and collective memory, that one which stabilises and enriches through history or through shared, intersubjective experiences. Or even to phylogenetic memory, as it seems that some pre-conceptual invariants, such as small numbers, are part of our inherited experience (see [Dehaene,1998], [Longo, 1999])

By this cognitive and historical formation of sense, meaning, as reference to space, or action, or to structures of language, that we later called "logical", or to other forms of knowledge, is at the core of the certainty, objectiveness and effectiveness of Mathematics; these are the final result of this constitutive processes towards conceptual stability.

In other words, Mathematics is based on the constitution of conceptual invariants, grounded of a variety of "acts of experience", distilled in praxis, by our action in the world, from movement in space to memory and displayed by language in intersubjective communication; these invariants are "what remains" once the "details" are erased; they are 
the common conceptual structure which explicitly expresses our relation to the phenomenal world. The permanent reference, while theory building of problem solving, to the networks of constituting meanings is the reason for their certainty, effectiveness and objectivity. The mathematician's intuition is the grounding of understanding into this network of meanings. Its analysis is an integral part of a foundational project and, as such, it cannot be only a (meta-)mathematical problem: it is a cognitive issue, which spans from Biology to History.

\section{Meaning and intentionality, in space and time.}

"Geometry ... is engendered in our space of humanity, beginning with a human activity" [E. Husserl, 1936].

The first locus for meaning are space and time. Well before any explicit or conscious representation, the first goal directed action is that of the living cell that moves in a direction, in order to maintain or improve its metabolism. And this a "meaningful action" and its meaning is at the core of life: it is meaningful, at the most elementary level, as it is part of a goal, of an intention (it is intentional ${ }^{5}$ ).

In order to understand this approach to meaning, we need to analyse the difficult entangling of finalism and contingency that is central to life. In contrast to inanimate objects, a living being needs to "interpret" the world, in order to live in it. This may be at the most elementary bio-chemical level of the cellular reflex or at the incredibly complex level of our brain. At each moment we need to interpret the environment relatively to our main aim: survival. It is the "finalistic contingency" of life that forces us to give meaning relatively to an aim (finalism), our unavoidable aim, but it does not need to be there and strictly depends on, but cannot be reduced to, the context (its contingency): each individual life or even that of a species, life itself is contingent. This paradigm is finalistic, but it stresses contingency, as no species, no living being would be alive if it did not have this aim at each instant of its life; yet, life and its specific realisations are not a necessary consequence of the "previous state of affairs" (they are contingent). Thus, by contingency I also mean "context dependence"; and the two meanings are related, as "depending on the context" is entangled with the lack of general necessity.

The relation to finalism interests us, since giving meaning to an incoming information is "inserting/contextualising that input" into or w.r.t. an aim, a goal, this is the main thesis

\footnotetext{
${ }^{5}$ I am broadening by this the notion of intentionality in Husserl, as the prevailing husserlian tradition restricts intentionality to a conscious activity: intentionality is the (conscious) "aiming at an object (of consciousness)" and this object is meaningful exactly because it is "aimed at", consciously (see, for example, [Lanfredini, 1994] or the many papers on this in [Petitot et al, 1999]). In view of the remarks below, I dare to expand Husserl's clear and robust notion, in a compatible way, I hope.
} 
here (a similar idea is briefly hinted also in [Bailly, 1991], a remarkable essay in philosophy of science). We have at each instant both inputs and aims, at least a major one, life. The living being, beginning with the most elementary form of life, interprets inputs by comparing them against its main aim: preserving or improving its metabolism. Thus, "meaning" is first given by how much the inputs helps or diverts from it. In particular, this is where begins our relation to space and time, as living beings, well before any symbolic notation can be detached from them. Human geometry is effective, because it begins with the action in space of the amoeba, or with the squid choosing the shortest path to hide behind a suitably large rock (see [Prochaintz,1997] and Longo's review, downloadable). These are the very early steps towards an attempt to organise space, up to the human proposal to make space intelligible: geometry. A proposal among others: we called "mathematical" the one focusing on invariants and conceptual stability.

Of course, these are just the very remote origin of our relation to the world: it is like a little stone in the enormous mountain that evolution and, later, human history have been adding on top of it. Between the "meaning" of a chemical stimulus interfering with its metabolism, for an amoeba, and the meaning, for us, of a ... mathematical proof, there is an abyss, two billions years wide. However, there is also the continuity of life, a "non differentiable" continuum perhaps, with sudden turns, as life is necessary to meaning: this is constructed, first of all, as interpretation w.r.t. life's implicit finalism, then as a network of mutual references, of "explanations", up to the reflective equilibrium of our (scientific) theories.

In summary, meaning is at the core of effectiveness, in Mathematics and in other forms of knowledge as well. That is, Mathematics is effective since it is meaningful, if one understands meaning as relation to "our active presence in the world", from the simplest action of the living cell up to our endeavour towards complex relational life and knowledge. Or the rich blend of them, as one cannot clear cut between the "meaning" for the individual cell and that for the human individual, made out of cells. In our brain, neurons react as cells to stimuli, but they do it as part of assemblies of neurones ([Edelman,1992]), which in turn are influenced by our unity as living beings and, thus, by our "external" action and intersubjective exchange. These levels are not "stratified", one on top of the other, the one below specified independently of the above, but they interact in a self supporting way (in an "impredicative" fashion, to put it in logical terms). Moreover, senses are far from being "input channels", their computational parody, but they are a dynamical interactive systems, where the "form" of the input cannot be detached from action and aims: sensorial inputs are always actively selected and structured, according to an ongoing interpretation, for the purposes of action. This yields the complexity and richness of meaning for humans, as the non additive sum of "meanings" from the living cell up to intersubjectivity in history. 


\subsection{From Euclid's "aithemata" to Riemann's manifolds}

I pause here to sketch again some historical and philosophical remarks at the base of the author's contribution to a project, a scientific analysis in progress, as this analysis of human meaning in Mathematics is a long term goal, even when "restricted" to phenomenal space and time (see [Longo et al, 1999]).

First, we react to space as living beings: distance is measured by movement, muscular thresholds, vestibular memory of rotations, [Berthoz,1997.]. Time is related to it by action. But how do we go from these pre-conceptual experiences to the mathematical concepts?

The interlocking of space and time contributes to give meaning to both of them: explicit metaphors for time refer to space and conversely, or we understand one in terms of the other and of our presence in them (see the "metaphors" in [Lackoff and Nunez,2000]). We perceive the symmetries of Physics (the reflections of light, crystals ...) and we give them a major relevance: they are "meaningful" to us, as symmetries shape our bodies and our lives, they underlie our actions and aims, towards pursuing life.

Our geometric proposals are shaped along symmetries, as well as along lines of least action, geodetics, a further relevant regularity of space. This is so, say, for Euclid's "aithemata" (requests), which are five practical constructions with least tools (ruler and compass), grounded on least paths and symmetries: they are surely not "axioms" in the formalist sense, as they are rich of meaning, as action or constructions in space.

The first axiom, for example, that is "draw a straight line from any point to any point", describes an action along a Euclidean geodetic, and so on so forth with three more. The fifth axiom describes the most symmetric situation when drawing a line on a plane, by a point distinct from a given line. More precisely, following Euclid's statement, consider, on a plane, a straight line $d$ cutting two straight lines $b$ and $c$. Then $b$ and $c$ meet exactly on the side where they form, with $\mathrm{d}$, two angles of sum less than $180^{\circ}$. Or, they are parallel exactly when, on both sides of $\mathrm{d}$, the sum of the angles is $180^{\circ}$. Why this geometric assumption should be "the most convenient" for understanding the space of every-day life, as many claim, Poincare' in particular, but not fully general for a physico-mathematical analysis, as we know since relativity theory (and as Riemann and Poincare' himself had conjectured, see [Boi,1995])?

There are here two phenomenal levels, which stress the internal generativity of Mathematics, once its conceptual tools are well established: the local and the global analysis of space. First, the local analysis of neighbouring distances, as the space of movement and local perception, and its extension to the Euclidean plane. At this level, Euclid's fifth axiom describes the most symmetric situation: if one assumes convergence of the two lines on both sides (Riemann), or many lines that would not converge on either side, even when the 
internal angles are different form $180^{\circ}$ (Lobachevskij), then many symmetries, on the Euclidean plane, are lost. That is, for the local-Euclidean analysis, the two non-Euclidean cases lose all symmetry axes orthogonal to the two lines, except one, as well as the parallel (central) axis of symmetry. In equivalent terms, if one draws, in a point, exactly one parallel to a given line on a Euclidean plane, then one obtains more symmetries than when following the two "possible negations" of the Euclid's fifth axiom, naively represented in the Euclidean plane. Euclid's geometry constructively proposes an organisation of physical space grounded on (planar) symmetries (and straight lines understood as light rays, according to [Heath, 1908])

Yet, there is another phenomenal level. This one focuses on the locality of the Euclidean manifold and suggests also a global look to space. This understanding of the world is constructed in a difficult intersubjective practice, through history: it is the passage from the Greek geometry of figures to geometry as a science of space, from Descartes to Gauss and Riemann. Along this path, man had to get used to manipulate actual infinity, a difficult historical achievement, far away from Euclid's understanding (see the fuzzy use of "apeiron", indefinite, in the fifth axiom and in the definition of parallel: "eis apeiron", "in apeiron" ..., a truly indefinite concept, in contrast to the perfect rigor of the other notions).

One major step towards this achievement, consisted in conceiving the convergence point of two parallel lines, "out there", into actual infinity, a necessarily global look to space. This is projective geometry: it provided an early, implicit, distinction between the local level of the figures of Euclidean geometries and a global level of a geometric space, which includes the point at infinity. Projective geometry is still compatible with the Euclidean approach, yet it is a relevant extension of the latter, largely due to the pictorial experience of the prospective in the Italian renaissance (this is when projective geometry was actually invented). The mathematical proposal grew out from the interaction with painting, a remarkable example of this singling out of mathematical concepts from our attempts to describe the world, even for very different purposes. By this, also, it became possible to conceive the phenomenal level of actually infinite planes, where one may have that remote convergence point of parallel lines. In summary, the geometrical experience is enriched by the proposal of a second phenomenal level, the global level of actual infinite spaces, beyond, but compatible with the local level of Greek geometry of figures. The infinite and absolute of Newton's spaces is a further development of this new conception.

Gauss and Riemann's analysis of curved surfaces is a dramatic change of view point. The idea is that the global geometric properties could differ from the local ones: ratio of distances could vary when enlarging figures. As a matter of fact, Euclidean geometry is the only one whose transformation groups (whose automorphisms) contain the homotheties (i.e., its local properties, such as ratio of distances or angles, are invariant w.r.t. arbitrary 
enlargements and their inverse): homotheties are not automorphisms in non-Euclidean geometries. In Gauss and Riemann's differential geometry, distance can be defined locally in an Euclidean fashion, by generalizing Pythagora's theorem to differentials (in a bidimensional manifold one may set $\mathrm{ds}^{2}=\sum \mathrm{g}_{\mathrm{ij}} \mathrm{dx} \mathrm{i}_{\mathrm{dy}} \mathrm{j}$ and this determines the local structure - the metric and curvature). As for the global structure, it is topology that matters, as the Cartesian dimension is a topological invariant (dimension is preserved exactly under topological isomorphisms), while (relative) distance is a local property (the metric structure is a local property).

As already mentioned, Riemann, in 1854, conjectured that the presence of physical bodies could be related to the local properties of distance, the metric: a remarkable insight towards relativity theory, as acknowledged by H. Weyl in 1921 (see [Boi, 1995]). Riemann (but Gauss and Lobachevskij as well) was explicitly working at a geometry of physical spaces. This stresses the relevance of his revolutionary proposal towards a way by which we organise space and understand the world (see [Bottazzini, Tazzioli, 1995]). A proposal grounded on "acts of experience", as Weyl would say, i.e. on a progressive formation of sense, through history (see [Weyl, 1927]) .

This change of perspective largely influenced our understanding of Physics, as we all know, since it modified the key "phenomenal veil", in the sense of Husserl, as the interface between us and physical space, and thus it modified the geometric intelligibility of it. NonEuclidean geometries, or, more generally, the treatment of space as a (riemannian) manifold, proposed a novel phenomenal level, as locus of the interaction between us and space. A new Physics is constructed over it: the effectiveness of the proposal is due to the fact that it is the very (mathematical) language for a new conception of space and time. It organises the world and generates the new objects of the physical reality; by this, it defines an understanding and helps to predict. There is no pre-organised reality that we perfectly describe, by miracle, by our independent tools (either formal or platonic Mathematics): these very tools are proposed while organising reality, in space and time, and trying to make sense of it, from Euclid to Riemann.

\subsection{More on symmetries and meaning}

As already mentioned, in Euclidean geometry, the local properties are extended for free, by homotheties, to the entire space; in particular, the local notion of parallelism is extended "indefinitely", by the (apparently) more symmetric situation (i.e., w.r.t. the naive Euclidean interpretation of the two negations of the fifth axiom). And symmetries are meaningful for us, as living beings: our own body is organised according to symmetries; we detect them very easily and use them regularly in action and pattern recognition ([Berthoz,1997], [Ninio,1991] and many others, for example in gestaltist approaches to vision). 
But how could physicists give up, in this century, such a meaningful and relevant physico-mathematical property as symmetry and prefer, in some contexts, non-Euclidean geometries? The point is that the newly constructed phenomenal level, the one which allows to conceive different "global" geometries, has still enough symmetries, from an algebraic point of view. Yet, how to count symmetries, as they are infinitely many? A sound way is to analyse the group of isometries. Now, this group, on the plane, is generated by the symmetries (as reflections) and it can be proved that there are isomorphisms (of different sorts: algebraic, topological) between these groups in the various geometries. Thus, from the new, subsequently generated mathematical point of view, that of algebraic geometry, one has that symmetries, in the different geometries, have a "similar" algebraic expressiveness. And then, physicists, when working at the abstract level of formal representations of space, may indifferently find more suitable, in order to describe space, one geometry or the other, according to empirical evidence, when possible. That is, as far as symmetries are concerned, there are no general-algebraic reasons to prefer one geometry to the other, at least not grounded on symmetries, while there may be empirical ones (the curvature of light in astrophysics, typically); yet, the Euclidean approach is the most obvious (convenient?) extension, by homotheties, of our local space of senses, with all its symmetries. These are the "evidences" behind Euclid's axioms.

Note instead that the mathematical descriptions of space in microphysics and in astrophysics are not closed under homotheties, so far: the geometry of quantum mechanics, the Euclidean geometry of "medium sized objects" and the spaces of relativity have properties that cannot be transferred one from the other by homotheties. There is no unified geometry for these three phenomenal spaces, a geometry invariant under homotheties; but there are good reasons, there are evidences which underlie each geometric proposals. As a matter of fact, the evidences for the non-Euclidean negations of Euclid's fifth axiom, are based on a peculiar "path through history", which we sketched above and which enriched our mathematical constructions: by this we could propose new physical experiences and describe a different understanding of geodetics, say (the light rays of Relativity). As a matter of fact, the concept of infinity, symmetries and their interplay in the distinction between local and global phenomenal levels, in the analysis of space, gave us a language by which could propose a new understanding of (the notion of) rigid body and light ray.

As for the notion of "evidence" above, the point we are trying to develop is beautifully hinted by Husserl: "the primary evidence should not be interchanged with the evidence of the "axioms"; since the axioms are mostly the result already of an original formation of meaning (Sinnbildung) and they already have this formation itself always behind them" [Husserl, 1936; p. 193]. Axioms, then, even the "meaningful constructions" by Euclid's, are not the bottom line of the foundational analysis: geodetics or symmetries, as meaningful 
aspect of our manifolded relation to the world, are "behind them", in Husserl's sense. And these properties of space and of our relation to space, do not depend on the specific geometry, but, in different forms, they are also "behind" the axioms of non Euclidean geometries, jointly to the other properties that Riemann, Poincare', Weyl and a few others begun to analyse: isotropy, continuity, connectivity (see [Boi, 1995]).

Thus, the formal, unintepreted axioms, Hilbert's style, are far away from founding Mathematics. Instead, they, in turn, are grounded on meaning, often to be made explicit, often necessary to the proof, as mentioned in the section 1.2, even for the most mechanisable of our mathematical theories, the arithmetic of integer numbers. These meanings relate Mathematics to the world, ground its constructions in it and, by this, turn Mathematics into a certain, objective, effective science. Intuition is the bridge that provides foundation, by "understanding", that is by embedding formal notations in a network of meanings; these are "behind" the constituting of conceptual invariants, as intentional selection of common elements, of bridges and analogies, interpreted for the purposes of aims, such as life, actions and human search for knowledge.

\section{Contours and stability.}

There is no split between mathematical constructions and the world, as we draw Mathematics, its "geometric or conceptual contours", on the "phenomenal veil", that is on the interface between us and the world. We ground it by this in regularities of the world, while singling out these very regularities and defining our own "self". The construction of objects, our singling them out, and of concepts, derives and gives meaning from and to the world. By these reasons, by this rooting of knowledge in the meaningful presence of our biological and historical life, by this cognitive presence of ours in the world, Mathematics is so effective for its purposes. Mathematics is, by definition, the collection of the maximally stable concepts that we can draw on the phenomenal veil, the invariants that we may transfer in many other forms of description of phenomena, exactly because of their stability and invariance or strong contextual independence. And we always need to single-out an invariant in order to constitute a (physical) object. Moreover, we simultaneously design and force a formalism from and onto reality: Mathematics is normative and generative, as it proposes rules and methods for deriving new concepts, new structures. These may then suggest a new understanding of the world, a new tool for "reading" it. Mathematics, thus, is effectively applicable to different contexts of knowledge: it forms the underlying texture of our representations of the world, by its very construction.

The point is that we actively single out objects and propose concepts. And we extend this "action" into autonomous mathematical constructions: the (more or less formal) 
mathematical developments are a purely mental extension, made in language and intersubjectivity, of the human praxis of generating stable (geometric or conceptual) contours. Of course, Mathematics also departs, by its internal methods, from its direct rooting in our understanding of the world, but these very methods derive their sense and are made possible exactly by our active and creative intuition, in the husserlian sense (see [Boi, 1998] for a clear understanding of Husserl vs Kant on intuition).

In other words, while determining our "spaces of humanity", we simultaneously draw the borders of "objects", we single out relevant contours, we understand while interpreting and naming. Our ego is constructed at the same time as the world of phenomena around us. Of course, there is a reality "outside there", which oppose "resistance and frictions" to our action, but phenomena are constituted in the interface between us and this unorganised "reality".

Mathematics plays a major role in this process, at least in Physics, or as soon as our action tends towards scientific or sufficiently general knowledge. Mathematics "singles out" contours of objects, by the drawings of geometry and, more generally, by conceptual shaping of images and ideas. Mathematics is the drawing of contours which do not need to be there.

Human vision is a good paradigm for this, as we do not "swallow" images passively, but they are (re-)constructed by active interpretations. In vision, some areas of our primary cortex reacts only to contours. But contours are not objects, they are singularities, in the mathematical sense, at the edge of bunches of wave length. We perceive these singularities and use them to isolate one object from the other, by deciding where and how to "cut" or delimit those inexisting lines. This is done in a continual interplay between incoming messages and interpretation: visual illusions tell us the permanent role of interpretation in vision, on the grounds of memory, interpolation, tridimensional reconstructions.

Consider, say, the names of colours, so history dependent, yet not arbitrary. It is a completely human and historical choice that of categorising colours, like separating blue from green, by giving, with a name first of all, "individuality" to this or that colour, marking borders in the "continuum" of wavelengths, between say "burnt siena" and "red amaranth" or even "blue" and "green" (Euclid, as all Greeks, had the same name for blue and green). Yet wavelengths are there, as well as our retinal receptors, which have "pigments" sensitive to three primary colours (they have excitement peaks corresponding to the wavelengths of red, blue and green): these colours, as parameters in a three-dimensional space (an example of a three dimensional manifold, mentioned by Riemann), allow the reconstruction of all possible wavelengths, but many other triples would do as well.

It would be interesting to know more about the role played, in the history of language, by those three primary colours that evolution has given us to reconstruct the others. They 
are like "pivots" upon which we build our mental categorisations and that probably drive our choices favouring some lengths over others, thus making non-arbitrary our categorisations. Because this is the point: scientific (and mathematical) reconstructions of the world are possible proposals and yet they are not arbitrary. Thus, the foundational issue is in singling out the phenomenal "pivots" on which, along history, we built up our forms of knowledge. As for geometry, and following Riemann, Poincare', Weyl, we referred to symmetries, isotropy, continuity and connectivity of space, regularities of action and movement, as "meaningful" properties. They are meaningful as they are embedded in our main intentional experience, as hinted above: life.

In Mathematics, then, we have been singling out conceptual contours, grounded on these "pivots" and regularities in the world, comparable to the three primary colours in the pigments of our retina. Then, we stabilised them in "abstract" (contextual independent, yet meaningful) geometric and linguistic invariants. More precisely, the core of the mathematical work is in turning the relations between invariants into norms and, then, using these norms to carry on further constructions (and proofs of further relations between constructions). This is the normative character of Mathematics: by Mathematics, we structure scattered phenomena by norms. Then, further mathematical structures extend the conceptual construction to more complex forms, built one on top of the other, interrelated by morphisms, which preserve the intended invariant (continuity say, for topological morphisms, operations for algebraic ones) $\ldots$ and this gives "categories of objects and morphisms"; then categories are related by "functors", which transform objects into objects and morphisms into morphisms. By continuing the category-theoretic metaphor, "natural transformations" relate functors and categories and so on so forth. Mathematics acquires then that typical autonomy from the world which singles it out from other forms of knowledge: it is grounded on a few cognitive and historical pivots and, once some invariants are stabilised by drawing and language, we use a variety of conceptual tools, a blend of many experiences (logical, formal, spatio-temporal ...), to constitute norms and derive new invariants, often far away from the ones we originally derived by our active, interpretative presence in the world.

\section{Microphysics and dynamics}

Physics has always been the privileged discipline of application for Mathematics. Indeed, Mathematics itself owes most of its own constructions to attempted descriptions of the physical world, beginning with Greek geometry, a dialogue with the world and with Gods at the same time. By this, geometry was detached by them from the "measure of the ground", an early process of "singling out" perfectly stable and invariant figures. Up to the 
infinitesimal calculus and Gauss-Riemann differential geometry, explicit attempts to describe movement and physical space. And further on, till the Mathematics of Quantum Mechanics, where the audacity in singling out inexisting but non-arbitrary contours reaches its highest level. "Each element ... must be prepared; it must be sorted; it must be offered by the mathematician. We then see to appear, in physical sciences, the opposition between descriptive and normative. The attributing of a quality to a substance was once of a descriptive nature. Reality had just to be shown. It was known as soon as it was recognised. In the new philosophy of science, we must understand that the attributing of a quality to a substance is always of a normative nature. Reality is always the object of a proof." [Bachelard, 1940; p.89].

As a matter of fact, in microphysics, we see nothing but some "crick-crack" or spots on measure instruments, which are far away from "phenomena". Then a mathematical theory is proposed, which gives unity to these "symptoms" by drawing a mathematical interpretation and, by this, by turning them into elements of a phenomenal description. Actually, even the physical measure instruments are constructed on the grounds of a theory, as they are just a practical explicitation of a theoretical hypothesis: we should measure this and that, in this way. Atoms, electrons, photons, gravitons ... are not there, they are "mathematical contours" which we single out by unifying a few signals. They are a way to propose non-arbitrary physical or conceptually stable borders. They are not "objects", yet they are as objective as our most robust theories of the world, since the physical world does "make resistance" and forces some signals towards us and the measure instruments we constructed. Physicists are ready to update them continually, even if, at each stage, the proposed invariants may be at the core of a relevant mathematized theory, often rich in applications.

This drawings of contours, these constructions of invariants and of perfectly stable conceptual entities are at the core of the applicability of Mathematics to inanimate matter, they actually are at the origin of Mathematics itself. Or, following Boi's interpretation of Weyl, "physics is but geometry in act" ... "so that the mathematical understanding of this world cannot be separated from the understanding of reality itself", [Boi,1998] (see [Connes,1990] for a geometric insight into Quantum Mechanics). Or, to put it otherwise, we understand Physics (movement, gravitation, non-locality, say) or we have it as "phenomena", by the very act of proposing a mathematical theory of space-time.

Of course, the effectiveness of the mathematical tool is relative to the interaction of an ongoing proposal and the various phenomenal levels: unifying phenomena is a major criteria for effectiveness. This proposal is an integral part of phenomena, but gaps are possible as we make "choices" while setting conceptual contours. These choices are not arbitrary, as they are grounded on incoming signals, on meanings, on accumulated, 
historical knowledge. The selection is made with reference, often, to other forms of knowledge, by implicit analogies and metaphors (such as the metaphor of the planetary system, say, to understand the atom). Thus, they may yield incomplete descriptions.

An example is given by the dynamical systems which are sensitive to initial conditions: we cannot predict completely they quantitative evolution by our mathematical tools. Their effectiveness then is limited, as predictability is a component of effectiveness, even if they give a better understanding of phenomena, by unification or explanation.

Of course, impredictability is not a matter of the world: there is no way to know whether the "physical reality" is "chaotic per se". The question only makes sense at the phenomenal level, the only accessible one, the actual interface between us, or our nonarbitrary proposals to organize the world, and "reality". As a matter of fact, God may know very well where the Earth will be in more than 100 millions year (see [Laskar,1990] for impredictability results on the solar system). In deterministic, chaotic systems, the only "fact" is given by an increase of "complexity" of some phenomena; but this understanding is already a theoretical proposal, an organized understanding. That is, impredictability shows up when something is said (dicere), or concepts are displayed, or they are proposed by a (living and thinking) conceptor: it is not a metaphysical truth. Impredictability requires an attempted prediction, while interacting with "physical reality" (or signals coming out of it); it concerns our mathematical attempts to organise these signals and it says that our attempts are "provably incomplete".

\section{Incompleteness in Mathematics and Physics}

One may draw then an analogy between the incompleteness results in formal theories (see sect. 2) and the one mentioned above, concerning dynamical systems. Let us first recall that the incompleteness results in mathematical logic, in formal arithmetic in particular, evidentiate a "gap" between the formal, theoretical level and meaningful mathematical structures: one cannot "remove the machinery" from proofs, a machinery which refers to transfinite ordinals or well-orderings, as constructions in mental space and time. In these cases, the normative structuring of Mathematics extends iteration and order, beyond phenomenal time and space, towards and by the concept of infinity. This is the nature of purely mental constructions, well beyond the finite, such as transfinite orders or infinite well-ordering. In a sense then, infinitary constructions in mental space and time may be understood as the subjective traces of intersubjective extensions of the objectivity of the phenomenal world, i. e. the are the "mental marks" of the objectivity we constructed in intersubjective, historical praxis, over basic regularities. The concept of actual infinity is the result of many historical conceptual constructions (theological, based on the projective 
geometry of renaissance painters ...). Its objectivity is obtained as an integration of "metaphores" (see [Lakoff\&Nunes, 2000]; but they are not just linguistic metaphores) and by the normative structuring of Mathematics, well beyond phenomena and leaves traces in our minds; it is extended by a blend of manifolded experiences (the metaphysics of infinity, say, played a major role in the constituting of the mathematical concept, from St. Thomas to Leibniz, see [Zellini,1980].)

The finitary formal approach, which does not include these meaningful structures, such as infinitary mental constructions (the well-ordering of the set of all numbers, say), cannot completely describe their properties, as it has been proved. In the case of arithmetic, the incompleteness is due to the fact that the well-ordering of standard numbers cannot be axiomatized in a finitistic-effective way, yet it is an absolutely clear and robust structural property of integer numbers, seen as actual infinity in mental spaces: when used in (human) proofs it yields formally unprovable results (see, for example, the proof of Friedman's Finite Form of Kruskal's theorem, quoted in $\S .1 .2$ ).

One century before Hilbert's wrong conjecture of the completeness (and decidability) of formal theories, Laplace had formulated a similar one, in mathematical Physics: in his opinion, the systems of (differential) equations could completely describe the physical world. More precisely, if one wanted to know the state of the world in a future moment, with a given approximation, than it could suffice to know the current state of affairs up to an approximation of a comparable order of magnitude. By formally computing a solution of the intended equations, or by suitable approximations by Fourier series, one could deduce (or predict or decide) the future status, up to the expected level of approximation.

Poincare', as a consequence of his famous theorem on the three bodies problem, proved that minor variations of the initial conditions could give enormous changes in the final result or, even, that the solutions could depend discontinuously on the initial conditions. Then, predictability, as "completeness w.r.t. the world" of a suitable set of formal equations, failed. These results, thus, and the subsequent work in dynamical systems, are the mathematico-physical predecessors and analogues of the many proof-theoretic incompleteness theorems, since Gödel. They set a limit to the effectiveness of mathematical tools in Physics, but they are also at the origin of beautiful and new mathematical theories, where qualitative predictions replace quantitative ones and where the "mathematical understanding" does not need to coincide with predictability (see [Thom, 1972]). Moreover, these theories, by forcing more geometry and topology into the prevailing analytical approaches to Physics, gave further richness and unity to Mathematics (see [Devaney, 1989] for a survey of the geometric approach to dynamical systems).

In conclusion, there is no absolute effectiveness of the mathematical tools, in Physics, but the constructed objectivity of Mathematics is grounded on an interaction with the world 
around us (and in us) that guaranties a relative effectiveness, though remaining often incomplete. No choice of a specific level of description, such as the mathematical one, given by some linguistic constructions and some geometric contours, may yield a complete representation of the richness of the universe we are embedded in as we have several and interacting forms of representation. Moreover, language and drawings are rich of our internal finalism and interpretation and cannot perfectly coincide with any "independent" physical reality. Their are reasonably effective because meaningful, i.e. because they are grounded in our cognitive being, as an ongoing process that constructs "reality" while living and interpreting it. Yet they are incomplete, by this very same reason: their objectivity is constructed by us, with our changing limitations.

Impredictability and incompleteness results are there to remind this to us, and to encourage the permanent invention of new methods and the construction of new "phenomenal levels". We have been able to do so throughout history by dramatic expansions of our tools or changes of paradigms: the birth of infinitesimal calculus and of non-Euclidean geometries are two of the most fantastic examples of this open-ended process, in Mathematics. The believe in "perfect completeness" or "absolute effectiveness" of the current mathematical tools (even of "formal" tools w.r.t. to specific mathematical structures, say), instead of the understanding of their relative completeness and reasonable effectiveness, may be misleading and may made us blind w.r.t. the growth of other form of knowledge, which may stimulate the change.

\section{Some limits to effectiveness: the phenomenology of life.}

The extension of the mathematical method to other sciences, where conceptual stability and invariance are not the main concern, is even less straightforward and sets further limits to its successes.

As a matter of fact, the richness of Mathematics is grounded on its unique invariance and stability: one may even define Mathematics as the locus of the maximally invariant and stable concepts that humans could propose, in their endeavour towards knowledge. Mathematics is normative in that this invariance and stability provide the norm, they are not (passively) descriptive. Its effectiveness, in Physics, is due to the essentially constructed (and mathematical) nature of "physical objects", as this is how we "single them out" (by mathematics). But, are mathematical invariance and stability, its (fully general) norms, at the core of Biology?

Consider, for example, the notion of neural synapses. Of course the biologist has to "define" it, as he has to communicate knowledge, propose a description. Yet, the notion of synapses does not need to be as stable as a mathematical concept, for many reasons. 
Synapses change in evolution and ontogenesis; they are dynamical; their behaviour is largely contextual and no fully general norm describe it, as the causal relation w.r.t. the context is less relevant that the finalistic organisation of the system of which it is part. Norms are very effective in explaining causes, much less in understanding the contingent finalisms of life. The ecosystems of life change continually the rules of the game. The underlying physico-chemical invariants are part of the phenomenon, but are not sufficient to describe it. Their analysis contributes to the explanation, but the phenomenal level of our relation to living beings is a different one. By this, Physics and Chemistry, and their invariant laws, are necessary to understand life, but they are not sufficient to derive its properties.

Clearly, we may change mind, in history, as for the description of an electron, as well: experiences may bring in new facts and suggest novel interpretations. But, in Biology, it is not just knowledge that may be revised, as in Physics and all empirical sciences, but stability, full generality of the "formal" description, repeatability of the experience, are less central than in Physics. What really matters are variation, non-isotropy, diversity, behaviours in an ever changing ecosystems. Again, one needs to write about and, then, to define the synapses, but, by the reasons above, a mathematical definition of it would not have the same interest nor relevance as the unavoidable and crucial or fully explanatory, mathematical description of a particle, in microphysics (see [Jacob,1970], [Bailly,1991], [Longo,1998], among others). For example, one may mathematically define a quark and derive (some) of its properties as theorems, to be later checked by experiences; a mathematical definition of a neural synapses in no way (or in minor, very specific ways) could give properties of the biological entity as "theorems", to be formally derived from the definition.

However, although "biological objects" may be hardly captured by the normative nature of the physico-mathematical description, one may consider another element of the biological phenomenon, which has no counterpart in Physics: functionality. It is possible, that the "function" of a living component, organ or being (in an ecosystem or in a compound form of life), may be more effectively described, by mathematical tools, than the "object".

The problem of effectiveness then is transferred to the analysis of the "right level" of invariance to attribute to functionalities, i.e. to propose an informative and effective level at which the function can be actually abstracted form contextual dependencies, or may be given the right level of dependence on them and, thus, stabilised in a mathematical description. The point is to find "what depends on what", or how much a specific function of life may be independent, say, from the "hardware" that realises it.

Indeed, this kind of problem is typical of Mathematics, in its own context. Category Theory, in Mathematics, beautifully centres it: one has to find the right category to work in, 
i.e. the structural properties that morphisms or isomorphisms are exactly meant to preserve. A category spells out the invariants that matter: this is the main reason for its conceptual superiority in the foundation of Mathematics w.r.t. Set Theory. Moreover functors relate categories to other categories, tell what must be added or forgotten to embed one into the other. Natural transformations relate functors and unify the various notions stabilised in and by functors and categories. But proofs as well, in Mathematics, require a close analysis of invariance. When proving a theorem for all real numbers, say, or an arbitrary algebraic structure, one may "use", in the proof, a generic real or a generic example. Then, at the end of the proof, the mathematician has to observe: "look, my proof only depends on the fact that this is a real or that is this kind of structure, no more no less is used". The task may be non obvious: it may happen that the proof "proves more" the statement, i.e. that less properties where required or that, under those hypothesis, more may be actually proved. Or, conversely, some implicit assumptions have been used. In both cases, the level of invariance proposed is the wrong one, too narrow or too large.

The research problem then, w.r.t. the uncertain effectiveness of Mathematics outside Physics, is to single-out some truly stable invariants, in Biology, say. Or, otherwise, to "adjust" Mathematics to more plastic conceptual constructions: instead of using well established methods and structures, with their usual conceptual stability, and work on the data provided by biologists, we should perhaps reconstruct concepts by interacting also with their methods, which are very different form those in Physics (see [Longo, 2001] for more on the distinction between concepts and structures in Mathematics).

As a matter of fact, it is very hard to transfer outside Mathematics the crucial theoretical praxis of the discipline, grounded on invariance. It is already very hard to apply it on the borderline of the mathematical activity, e.g. with reference to its applications, as we so often slipped into metaphysics: the relevance and stability of a proposal was confused with an absolute. So, for two thousands years, we were told that Euclidean geometry was "absolute", that it perfectly coincided with physical spaces, independently of any context or assumption, to physical space "per se". Similarly, Cantor-Dedekind's construction has been seen as "the continuum" of space and time. Closer to our times and to the problem we are discussing here, Turing Machines have been presented as the mathematically invariant definition of (human) reasoning, as discussed next.

\section{Thought as a function}

In 1935-36 the many formal approaches to computability (Herbrand-Gödel, Church, Kleene, Turing ...) were shown to be equivalent. The everybody exclaimed: we have an absolute. We coherently defined deductions as computations, independently of the 
formalism, machine or ... whatever implements it. May it be a Turing Machine, a mechanical (or, later, digital) computer, a set of formal rules ... provided that they contain certain features (as described by the partly informal notion of "algorithm"), then they all compute the same class of functions, the general recursive ones (this is the so called Church Thesis). And there comes the metaphysical slip: since these functions, as deductions, describe the "act of effective reasoning" (the "human computor in the act of deducing", to put it in the words of Gandy, a student of Turing) we have got the universal notion, the invariant defining human intelligence, in a complete and effective fashion. This is the so called "functionalist" approach to human mind.

We all know, since then, the many failures of Strong Artificial Intelligence, and the successes of many of its more modest "sub-programs" (the interactive expert systems and theorem provers and much more, which did not assume the full generality of the strong claim). As well as the successes of Computer Science, which is a "science" exactly because the "art of programming" is independent from the machine. Programs must be portable, this is the key motto of programmers. Programming languages must be "universal", i.e. compute all recursive functions and being transferable from a machine to another. Both key points are grounded on Turing's remarkable idea of distinguishing software from hardware. But even operating systems, as implementation of Universal Turing Machines, are transferable: so, when a machine dies (a new technology is born) one may take its operating system, its programs and date base and transfer it on another. This practice of "metempsychosis" is a remarkable technology and is at the core of Computer Science. Yet, some proposed for decades this model of computing, its perfect mind/body dualism, as a model for human intelligence (without necessarily being Indus).

The mistake again was to believe that "intelligence" may be grounded on formalised "laws of thought", as rules independent of meaning. Meaning as reference first to the "hardware" that implements it, our living and historical brain, and which is rich of the finalistic contingency of life we mentioned above. By this, the proposed level of invariance was the wrong one. The objectivity and generality of reasoning is instead grounded on that very peculiar hardware which is our brain through history, i.e. our human brains interacting by language and action with the world and among them. Its invariance is due to the common biological and cognitive roots and, later, to intersubjective exchange, which allows to focus on what is "stable" as shared with other humans, in an enlarging communicating community. Stability of (mathematical) reasoning, though very high (indeed maximal, among our forms of knowledge), is not an absolute and it is the result of a process towards invariance. It is very hard to spell out, in mathematical terms, which are the constitutive invariants, underlying this very "function", human thought, which produces invariants.

The irony is that even machines now do different things than that "absolute" proposed in 
the '30s. Distributed, concurrent, asynchronous computing uses open systems, working with evolving operating systems and data base, with no absolute time (a crucial physical difference w.r.t. Turing Machine). They perform very different tasks, in some cases, not even vaguely comparable with those of sequential computing with an absolute clock (see [Monist, 1999]). And we still do not have a sufficiently good mathematical description of these novel computer systems, which Physics and engineering have been giving us.

Yet, the "functionalist" approach to human thought still presents that wrong level of invariance (the "laws of thought" do not depend on life, are formal and, thus, they may be implemented on a Turing Machine) as the core of cognitive analyses.

This shows the difficulties in transferring the normative analysis of Mathematics from "objects" to "functions", when dealing with the phenomena of life and history. It is hard, if ever suitable, to single-out living individuals, mathematically; it is a non-obvious scientific challenge to extend the reasonable effectiveness of Mathematics to "functions", from the simplest living structure (e.g. the internal and external topology of a cell) to those which span life and history, such as human intelligence.

\section{Brain plasticity and neural nets.}

A more recent mathematical approach to mental functions has been grounded on the idea of "interactive net of formal neurones". These theories are beautiful physical-mathematical models, a qualitative change w.r.t. those that rely on mathematical-logic descriptions, such as Turing Machines or alike, based on the assumption of a universal "computational logic". Following McCullogh, Pitts and Hebb, neural nets on the contrary are inspired by the plasticity of the brain and aim at modelling this crucial aspect of it. In contrast with the functionalist theories, rather than asserting that the "hardware" which realises the thinking is irrelevant (Turing machines, systems of balls and marbles, computers or brains "are all the same" for the functionalist), the mathematical theory of neural nets assumes as essential a biological property of that specific hardware which is "the (human) brain", plasticity. In other words, instead of starting with a logical analysis of (mathematical) deduction and defining it to be an analysis of thinking, the connectionist hypothesis (such is the name for the theoretical proposal of neural nets) stresses a fundamental property of the brain, the plasticity of its electrical connections, and turns it into Mathematics. And this is a Mathematics very rich in powerful tools: "spin-glasses" and methods from statistical Physics, based on the Mathematics of dynamical systems, one of the most modern and powerful instruments of analysis in contemporary Physics.

But, at this point ... one forgets the original anti-functionalist project, the one which tries to develop and is prompted by the biological reality of the brain. One forgets that 
neurones are alive and have behaviours which are not always independent of their individual unity, let alone of their context. Indeed the brain plasticity itself depends on a number of causes. The first set of such causes is the spatio-temporal nature of the electrical message, its geometry. The second is the ability to prime "cascades of chemical reactions", which induce changes in synaptic structure. Third, the huge number of elements which regulate the chemical reactions within cellular and extra-cellular liquids. And still more causes, not yet well understood, including the tridimensional geometric structure of the proteins exchanged by the synapses.

The connectionist proposal seems to mutate into the following: it does not matter how brain plasticity is achieved so long as it is plasticity; the formal nets will simulate it by their very refined Mathematics of electrical connections of continuous weight. But then as soon as we start drifting away from functionalism, have we immediately gone back to it? We end up simulating with electrical circuits only one function, the one implemented by neural plasticity as variation of electrical conductivity, even if the real communication, along synapses, is also bio-chemical, it uses the convection of liquids etc.. At a certain point then one does not refer any longer to the structural characteristics of the brain, or forgets many of them which might be crucial but are not considered as such (fluids, bio-chemistry, ... ); again, we are back to thinking that "machines" are interchangeable. The subject then develops, driven by its internal methodology which again bears the physical-mathematical imprint.

All this is very interesting, since new questions can be asked to the biologist, and some specific situations simulated. But, mostly, formal neural nets suggest an original way of building extraordinary new machines, very different from digital computers. Still I think that physicists and mathematicians working with neural nets should not present their theory as a cut and dried "Theory of Brain": as we have said, the electrical signal along the axon is very important for understanding brain functions, but there are other factors which play a relevant role and "boundary phenomena" between membranes, Chemistry and liquids have their relevance too: the synaptic connection is far from transferring only electrical information, as it was once believed. Moreover, everything is coherently managed by that living cell, the neurone, which has its own well defined and aggressive individuality. This is killed if it is "conceptually dissected", if one focuses on a single physical phenomenon, the threshold electrical calculus. Moreover, this cell is immersed in contexts which are themselves alive and full of connections, which have their own unity and intentions (as aims of life), that are killed off by the "cutting plane" of the mathematical formalisation of the electric signal.

As I already stressed, this is the main reason why modelling in Physics differs qualitatively from that in Biology. Today, for instance, to shape a new kind of wing for an 
aeroplane one can often avoid experimenting in a wind-tunnel. Computer simulation of fluid dynamics has now reached a very high degree of precision and reliability. It is, in fact, an important element for cost-cutting in aeroplane construction. The mathematical description of that physical reality, namely the découpage of the "physical outlines", of the key parameters of the phenomenon and their electronic elaborations, are a good enough approximation for this practical issue. In this case the mathematical theory, even if at a level of approximation and formally incomplete, is an effective "theory" of the physical phenomenon.

On the contrary, there is a qualitative difference when trying to give a theory made of physical and mathematical invariants, which should capture the dynamics of living brains, say; or to propose a "theory" of the biological phenomenon, an effective one for simulating and forecasting the activity of the brain, beyond some very restricted aspects (mostly related to very small numbers of neurones). This is because the few mathematisable aspects will be conceptually isolated from the living whole, and one would work on them using one's own methods of conceptual stability; yet, it is that whole that contains an interconnected network of individual and global aims and intentions. As said in $\S .3$, intelligent behaviour is meaningful for this.

Certainly one must continue working at the mathematical modelling of biological phenomena, but keeping clearly in mind the limits of this approach. And the same to be able to talk productively to biologists (I have seen biologists and physicists with great difficulties in understanding each others on the theme of modelling and neural nets). In particular, in order to progress one must always remember that there is a qualitative difference between mathematical simulation in Physics and in Biology; a difference which I have tried to single out with these observations on "découpage", as a mathematical practice to isolate invariance, so crucial and effective in Physics, but rather unsuitable when transferred on the unity of living beings and their ecosystem.

Moreover, I wish to add that prudent researchers in this area, such as Hertz, Krogh and Palmer [Hertz,1991], admit that these theories have taken only one or two ideas from neurobiology, and do not make any pretence of giving a "mathematical model" of the brain. It is instead possible that these studies, with their autonomous practical and mathematical developments, might one day provide us with formal "neural" machines that can be even more revolutionary than those which have already changed our life: digital computers. As already mentioned, also Turing Machines were considered by many as the "ultimate" model for human reason, an absolute: they served to a, perhaps, more significant purpose, as they gave us brand new forms of elaborating and exchanging information. Computers are used for some fast numerical computations, impossible to man, or, mostly, for word-processing and world-nets of data, fantastic achievements, that do not even vaguely resemble our 
mental activities, but enrich them enormously. Good science is worth pursuing, when technically deep, even if the early philosophical project is basically wrong: the indirect fallout may be as amazing as unexpected. Subsequent philosophical reflections may help to revise it, or in inventing new research directions or, at least, in further understanding the world or our descriptions of it.

\section{Conclusion}

In this essay, I tried to delineate the "cognitive" reasons for the successes of Mathematics in Physics: Mathematics is drawn, simultaneously to Physics, on the phenomenal veil, i.e. on the very locus where we apprehend and (re-)construct physical phenomena, as living and historical beings. Its internal generative character, by norms and invariants, goes often further and independently of the relation to Physics; or, even, it may give, sometimes, indirect or unexpected new tools for drawings contours to novel physical phenomena. Thus, its effectiveness is contingent, as much as life itself, since it is first grounded in our active relation, as living beings, to space and time, by language and by all forms of intersubjective communication (gestures are not irrelevant in communicating Mathematics). Mathematics then may be described as a three dimensional space, as hinted in $\S$. 1.3, since invariants of space, of language and of formal computations interfere in a synergetic way while generating mathematical concepts and proofs.

Mathematics grows along with the reasonableness of History, which made us construct models of the physical world, since Greek figures of space, while creating the key structures of Mathematics or its very language. Yet, the effectiveness of extensions of Mathematics' fruitful paradigm to Biology is largely reduced, let alone to other disciplines where relational human activity is grounded in but go well beyond biological life.

When departing from the analysis of the causal and local or elementary interactions in Physics, the developments of mathematical tools must be done with a similar simultaneous attention not only to the facts and data of Biology but also to its own methodology, so indebted to finalism and global phenomena. As a matter of fact, in a two-ways interaction, the phenomenal level on which we draw Mathematics may change dramatically and it may require great changes in the mathematical methods, as least as relevant as the invention and use of actual infinity or of non-Euclidean geometries.

Of course, the switch from the analysis of "objects", in Biology, to that of "functions" has been at the core of a remarkable revolution. Morphogenesis is in part a consequence of this change of perspective: the analysis of singularities and fractals provided original tools for it (see [Thom,1972] and the many writings on Mathematics in Biology), yet the underlying physico-mathematical paradigm, upon which these ideas were born, still leave 
many biologist unsatisfied.

I have been choosing above the most complex of the "biological activities", human thinking, as an example of a "function". This is probably not fair, as the reasons for its complexity go well beyond Biology, in view of the role in it of human intersubjectivity, through history. Yet, at all levels of complexity, as soon as we examine functions of life, it seems particularly hard to isolate mathematical invariants, to find the right "category" and mathematical structures. This is largely due to the systemic unity, contingency and finalism in biological phenomena.

For these reasons, in [Longo,2001], it is suggested that, in order to gain in effectiveness, the interaction with Biology should begin by the analysis of the conceptual and even preconceptual constructions of Mathematics, which precede or underlie the explicit mathematical proposal for a structural invariant. We shouldn't only try to use well established or autonomous mathematical tools in order to contribute to Biology, but we should rediscuss foundational issues in Mathematics with reference to biological experiences (see [Longo,1998], [Longo et al., 1999]).

In conclusion, Mathematics does not capture "the essence of the world", even not in Physics, as we only interact with physical reality at the phenomenal level, where we "draw" the structures of phenomena exactly as mathematical objects, with no pretence to understand essences. Let alone in Biology where the unity of individuals, the interactions with the ecosystem and their contingent finalism appear instead as an "essence", well independent of us. The kantian paradigm shows here how linked it was to the peculiar relation between Physics and Mathematics.

In any case, though, Mathematics is as effective as it may be human language, as a tool for communicating. Human language is very effective, as we understand each other very well, but it is not unreasonably effective, nor "complete" in any sense. We need gestures, smiles, caresses, we need to hit or touch each other and make love to communicate more fully what language, in many situations, cannot express. Moreover, each human historical language is incomplete relatively to the others (and thus, it is "essentially" incomplete), as the following example may suggest.

A friend of mine, a French sinologue, had to translate a classic Chinese poem. This poem described a river running through a forest. In each ideogram there was a fragment of the ideogram that evocates the notion of fluidity, of running water ... . The visual, pictorial, impression was (reportedly) fantastic and it was an essential part of the poetic communication. This was clearly lost in the oral communication and, a fortiori, by the translation in an phonetic writing. Dually, it is almost impossible to translate in Chinese our complex temporal constructions, such as the past of the future or the future of the past; yet, both Chinese and our languages are very "effective" in describing the world. The universal 
and complete language of all possible expressions is a wrong dream as much as the universal (and complete) system of (mathematical) signs for all sciences. And this is fortunate, as it confirms the richness of the world and of our tools to understand/organise it.

This is why we invented autonomous conceptual (and linguistic) tools, w.r.t. Mathematics, for the analysis in Biology or, say, in History. Indeed, each method has some mathematical aspects, the mathematizable fragments. For example the morphology of a jelly-fish, which is shaped like a drop of milk falling into water (an old example recalled by R. Thom), or the spots on some fours whose distribution is optimal, according to some geometric criteria (more work in morphogenesis, since Turing), are beautifully mathematisable fragments of live; indeed, they are "physical aspects" of life. Similarly the physical structure or the "geometry" of the visual cortex may require some relevant Mathematics in order to be analysed closely. Yet, the biological phenomenon is also elsewhere: how comes, say, that this forms are genetically stabilised and are reproduced in offsprings? Or, how to analyse the crucial redundancies in evolution and even in ontogenesis, which are so unrelated to the optimal path or geodetics of mathematical Physics? By which path through evolution the "double jaw" of some reptiles of 200 millions years ago is potentially the hears of birds and mammals (the "latent potentials" in Gould's analysis)? The intended structures and concepts are so dynamical in evolution, that any mathematized focus on their conceptual stability and invariance would not be the most relevant aspect, as it necessarily is in Mathematics.

On one hand, then, it is possible that some fragments of life may be (increasingly) understood by Mathematics, but dually Mathematics owes greatly also to the other forms of description and knowledge, which do not need to be reducible to it. It contains some elements of these forms, which actually contribute to its foundation, as it is rooted also in them or, better, it possesses some common roots, the cognitive ones. And this is one of the reasons for the effectiveness of Mathematics.

Once more, this is not a vicious circle, but the virtuous spiral of the open and dynamical system of our forms of knowledge, if we reconstruct its unity as a network, not as a fake ultimate system of axioms which explains or "covers completely" everything, by formal derivations (the "fake wooden frame" to which refers Weyl in the introduction to Das Kontinuum, see the English translation; Weyl's anti-formalist stand is even more strongly presented in his posthumous [Weyl, 1985]).

The project then is in acknowledging first the differences in languages and methodologies, as well as their internal limitations, as for effectiveness, and then try to enrich them by interaction and, possibly, by singling out the common cognitive roots of our different conceptual constructions. Fortunately, these constructions, including Mathematics, are not God given, nor perfect and static platonic realms, but human and "plastic", as much 
as our interacting brains: thus we may invent better ones, as we did very often along history, and then unify them by cross explanations and mutual influences or translations. We may follow new meaningful aims, which may lead us to propose entirely novel concepts and ideal structures.

\section{References}

(Preliminary or revised versions of Longo's papers are downloadable from http://www.di.ens.fr/users/longo ).

Bachelard G. La Philosophie du non, PUF, 1940.

Bailly F. L'anneau des disciplines, n. spécial de la Revue Internationale de systémique, vol. 5, n. 3, 1991.

Berthoz A. Le sens du mouvement, Od. Jacob (english transl., Harvard U.P.), 1997.

Boi L. Le problème mathématique de l'espace, Springer, 1995.

Boi L. The role of intuition in Kant and in the modern Mathematics and Physics, Workshop on Kant's Theory of Intuition, Dortmund, 1998.

Boole G. The laws of Thought, 1854 .

Bottazzini U., Tazzioli R., Naturphilosophie and its role in Riemann's mathematics, Revue d'Histoire des Mathématiques n. 1, 3-38, 1995.

Connes A. Géométrie non-commutative, Paris, 1990.

Dehaene S. The Number Sense, Oxford UP, 1998.

(Review/article downloadable from http://www.dmi.ens.fr/users/longo.)

Devaney, R. L. An Introduction to chaotic dynamical systems, Addison-Wesley, 1989.

Edelman G. The matter of Mind, 1992.

Frege G. The Foundations of Arithmetic, 1884 (english transl. Evanston, 1980.)

Galileo G. Dialoghi sopra i due Massimi Sistemi, II, 1632.

Girard J.-Y. "Linear Logic" Theoretical Comp. Sci., 50 (1-102), 1987.

Girard J.Y., Lafont Y., Taylor P. Proofs and Types, Cambridge U. Press, 1990.

Gödel K., Nagel E., Newman J., Girard J.-Y. Le théorème de Gödel, Seuil, 1989.

Harrington L. et al. (eds) H. Friedman's Research on the Foundations of Mathematics, North-Holland, 1985.

Heath T.L. The Thirteen Books of Euclid's Elements, Cambridge Univ. Press, 1908.

Hertz J., Krogh A. and Palmer R. Introduction to the Theory of Neural Computation, 1991. 
Husserl, The origin of Geometry, part of Krisis, 1936.

Jacob F. La logique du vivant, Gallimard, 1970.

Lakoff, G. and Nunez, R. Where Mathematics Comes From: How the Embodied Mathematics Creates Mathematics, New York: Basic Books, 2000.

Lambert D. Structure et Efficacité des intéractions recentes entre Mathématiques et Physique, Thèse de Doctorat, Louvain-la-Neuve, 1996.

Lanfredini R., Husserl: la teoria dell'Intenzionalità Laterza, 1994.

Laskar J. "The chaotic behaviour of the solar system", Icarus, 88: 266-291, 1990.

Longo G. "Mathematics and the Biological Phenomena" in International Symposium on Foundations in Mathematics and Biology: Problems, Prospects, Interactions, (Basti ed.) Pontifical Lateran University, Vatican City, November, 1998.

Longo G. "The mathematical continuum, from intuition to logic" in Naturalizing Phenomenology: issues in comtemporary Phenomenology and Cognitive Sciences, (J. Petitot et al., eds) Stanford U.P., 1999c.

Longo G. "Mathematical Intelligence, Infinity and Machines: beyond the Gödelitis" Invited paper, Journal of Consciousness Studies, special issue on Cognition, vol. 6, 11-12, 1999a.

Longo G. "The Constructed Objectivity of Mathematics and the Cognitive Subject" Quantum Mechanics, Mathematics and Cognition (M. Mugur-Schachter ed.), Kluwer, 2001.

Longo G. "Mémoire et Objectvité en Mathématiques", Le Réel en Mathématique, colloque de Cérisy, 1999 (à paraître).

Longo G., Petitot J., Teissier B. Géométrie et Cognition, research project, downloadable from http://www.dmi.ens.fr/users/longo , 1999.

Philosophy of Computer Science, special issue of The Monist (G. Longo ed.), vol. 82, n.1, Jan. 1999.

Ninio J. L'empreinte des sens, Seuil, Paris, 1991.

Piazza M. Intorno ai numeri. Oggetti, proprieta', finzioni utili. Mondador, 2000.

Prochiantz A. Les anatomies de la pensée, Odile Jacob, 1997. (Recensione/articolo downloadable da http://www.dmi.ens.fr/users/longo.)

Riemann B. "On the hypothesis which lie at the basis of geometry", 1854 (english transl. by W. Clifford, Nature, 1873; trad. italiana di R. Pettoello, Boringhieri, 1999).

Shanker S. Gödel's Theorem on focus, Croom Helm, 1988.

Tazzioli R. Riemann, Le Scienze, Aprile 2000.

Thom R. Stabilité structurelle et Morphogénèse, Benjamin, Paris, 1972. 
Zellini P. Breve Storia dell'Infinito, 1980.

Waismann F., Wittgenstein und der Wiener Kreis, Frankfurt a. M., Suhrkamp, 1967 (Waismann's notes: 1929-31). (English translation: Wittgenstein and the Vienna circle : conversations recorded by Friedrich Waismann, Barnes \& Noble Books, New York, 1979)

Weyl H., "Uber die Definitionen der mathematischen Grundbegriffe", Math. naturwisss. Blatter, 7, 1910.

Weyl H. Das Kontinuum, 1918.

Weyl H. Raum, Zeit, Materie, 1918

Weyl H. "Comments on Hilbert's second lecture on the foundations of Mathematics." [1927] in van Heijenoort J., From Frege to Goedel, 1967.

Weyl H. Philo. of Math. and Natural Sci., 1927.

Weyl H. Symmetry, Princeton University Press, 1952.

Weyl, H. "Axiomatic Versus Constructive Procedures in Mathematics." (Edited by T. Tonietti) The Mathematical Intelligence Vol. 7, No. 4, Springer-Verlag New York, 1985.

Wigner E., "The unreasonable effectiveness of Mathematics in the Natural Sciences" Comm. Pure Applied Math., XIII, 1-14, 1960.

Wittgenstein L. Philosophical Remarks, translated into English by G. E. M. Anscombe, Barnes \& Noble, New York, 1968. 